\title{
Nanostructured Systems Improve the Antimicrobial Potential of the Essential Oil from Cymbopogon densiflorus Leaves
}

Janaína B. Seibert, ${ }^{\dagger}$ Juliana S. R. Viegas ${ }^{\dagger}$ Tamires C. Almeida,${ }^{\dagger}$ Tatiane R. Amparo, ${ }^{\dagger}$ Ivanildes V. Rodrigues, ${ }^{\ddagger}$ Juliane S. Lanza, ${ }^{\S}$ Frédéric J. G. Frézard ${ }^{\S}$ Rodrigo D. O. A. Soares,${ }^{\perp}$ Luiz Fernando M. Teixeira, ${ }^{\|}$Gustavo H. B. de Souza, ${ }^{\dagger}$ Paula M. A. Vieira, ${ }^{\nabla}$ José M. Barichello, ${ }^{\mathrm{O}}$ and Orlando D. H. dos Santos ${ }^{\dagger, *}$

${ }^{\dagger}$ Departamento de Farmácia, Universidade Federal de Ouro Preto, Ouro Preto, Brazil.

* Departamento de Farmácia, Universidade Federal de Juiz de Fora, Governador Valadares, Brazil.

${ }^{\S}$ Departamento de Fisiologia e Biofísica, Universidade Federal de Minas Gerais, Belo Horizonte, Brazil.

${ }^{\perp}$ Núcleo de Pesquisas em Ciências Biológicas, Universidade Federal de Ouro Preto, Ouro Preto, Brazil.

॥ Departamento de Análises Clínicas, Universidade Federal de Ouro Preto, Ouro Preto, Brazil.

$\nabla$ Departamento de Ciências Biológicas, Universidade Federal de Ouro Preto, Ouro Preto, Brazil.

${ }^{\text {o }}$ Departamento de Farmácia, Universidade Federal de Pelotas, Pelotas, Brazil. 


\section{RESULTS}

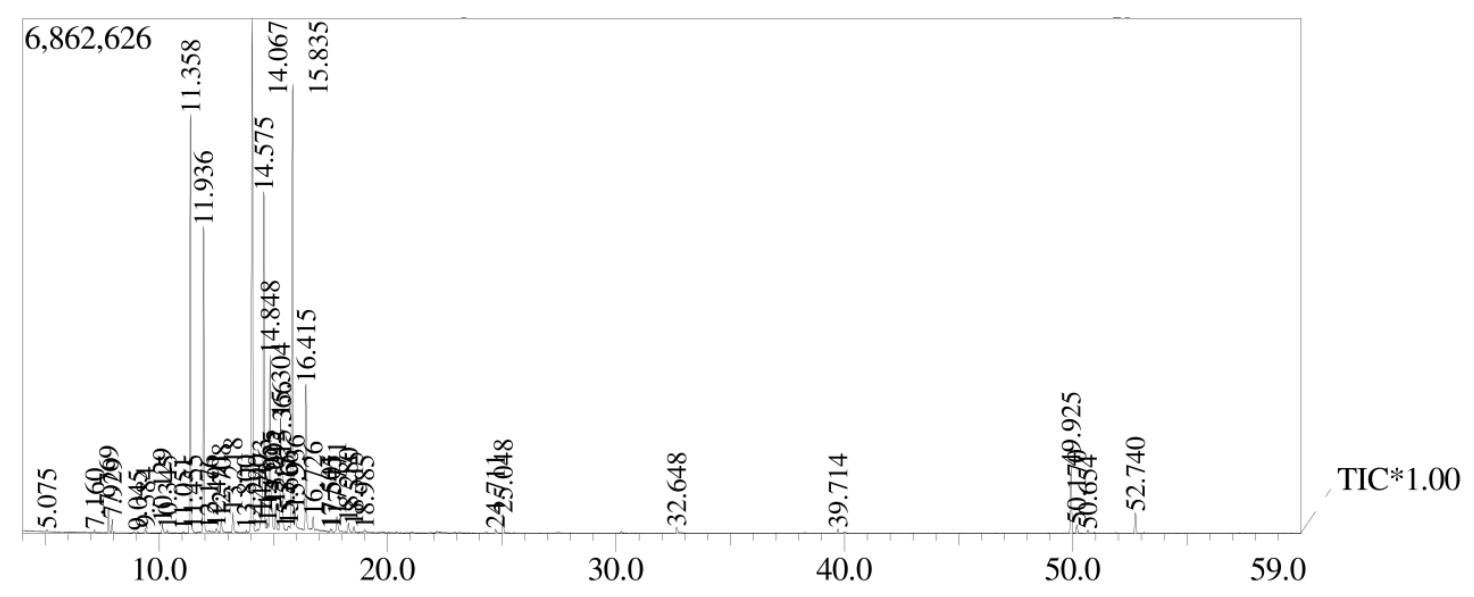

Figure S1. Chromatogram of essential oil from Cymbopogon densiflorus leaves with a maximum peaks relating to monoterpenoids.

\section{EXPERIMENTAL SECTION}

\section{Determination of nanoparticle size}

a) Dynamic Light-Scattering (DLS): The mean particle size of the formulations was determined by photon correlation spectroscopy using a Zetasizer (Malvern Zetasizer Nano model series - Nano ZS). This method allows the determination of the mean diameter of the particle and the polydispersity index (PDI), which is a dimensionless measure of the broadness of the particle size distribution. To estimate the particle size at room temperature, formulations 100 fold in ultrapure water (MilliQ Millipore). The incidence angle of the laser in the sample cuvette was $90^{\circ}$. The median and standard deviation analysis were obtained by triplicate for different times $(0,1,7$, $14,21,28$ days $)^{1}$.

b) Asymmetrical-Flow Field-Flow Fractionation (AF4): AF4 fractionation of the formulations was performed on a Postnova Analytics (Landberg, Germany) AF2000 MT AF4 system ${ }^{35}$. The fractionated sample was detected using a PN3211 ultraviolet (UV) detector with absorbance at $254 \mathrm{~nm}$, the gyration diameter $\left(\mathrm{D}_{\mathrm{g}}\right.$ ) of the fractions were determined with a PN3621 multiangle laser light scattering (MALLS) detector with a $532 \mathrm{~nm}$ laser $\left(7^{\circ}-164^{\circ}, 21\right.$ angles $)$, and the hydrodynamic diameter $\left(D_{h}\right)$ with a 
Zetasizer (Malvern Zetasizer Nano model series - Nano ZS), in series. The liquid carrier was $10 \mathrm{mM} \mathrm{NaCl}$ in Mili-Q water filtered $(0.1 \mu \mathrm{m})$. The formulations were diluted at 1:30 in the carrier liquid. The $\mathrm{D}_{\mathrm{g}}$ and $\mathrm{D}_{10}, \mathrm{D}_{50}$ and $\mathrm{D}_{90}$ distribution parameters were determined using the intensity of the UV signal as the value of concentration for each fraction relative to the total sample ${ }^{2}$.

c) Nanoparticle Tracking Analysis (NTA): The size distribution and the particle number density (number of particles per $\mathrm{mL}$ ) were also investigated by nanoparticle tracking analysis (NanoSight NTA $3.2 \mathrm{Dev}$ Build 3.2.16). This technique is based on the Brownian motion of the particles, recording frame-by-frame the diffusion of individual points of light scattered by each particle illuminated by an infrared laser beam. The diffusion coefficient of the particles in the medium is used to access the size distribution and particle number density after the video processing. The formulations were diluted in ultrapure water $\left(1: 10^{4}\right)$ and inserted into the viewing chamber of the equipment to video recording. The analysis settings to process the video were: 2 of Threshold, 15 of Gain, Auto Blur and Brightness at $25^{\circ} \mathrm{C}^{3}$.

\section{REFERENCES}

(1) Venkateswaran, S., Henrique Dos Santos, O.D., Scholefield, E., Lilienkampf, A., Gwynne, P.J., Swann, D.G., Dhaliwal, K., Bradley, M. J. Mater. Chem. B 2016 4, 54055411.

(2) Carneiro, S.P.; Carvalho, K.V.; Soares, R.D.; Carneiro, C.M.; Andrade, M.H.G.; Duarte, R.S.; Santos, O.D.H. Coll. Surf. B: Biointerfaces. 2019, 175, 306-313.

(3) Brum, T. L.; Fiel, L. A.; Contri, R. V.; Guterres, S. S.; Pohlmann, A. R. J. Nanosci. Nanotechnol. 2015, 15, 773-780. 